\title{
Management of a Child vs an Adult Presenting With Acral Lesions During the COVID-19 Pandemic: A Practical Review
}

Rebecca Candler Clawson, BS; Mika M. Tabata, MD; Justin M. Ko, MD, MBA

\section{PRACTICE POINTS}

- Children with chilblainslike lesions generally have a favorable prognosis. As lesions self-resolve, treatment should focus on symptom management and education.

- In children with chilblainslike lesions and no systemic symptoms, further workup for coronavirus disease 2019 (COVID-19) is not necessary for the care of the individual patient.

- In adults with acral lesions, it is important to distinguish between chilblainslike lesions, true acral ischemia, and retiform purpura. Chilblainslike lesions have been associated with mild COVID-19 disease, whereas acral ischemia and retiform purpura have been associated with severe and fatal disease.

- Biopsy and COVID-19 testing should be obtained in adults if there is diagnostic uncertainty or if there are worsening symptoms.

During the coronavirus disease 2019 (COVID-19) pandemic, there has been a rise in the diagnosis of acral lesions, including chilblainslike lesions, ischemia, and retiform purpura. Understanding the differences in presentation and severity of illness between children and adult patients is important for physicians to understand risk stratification and management of these lesions. We reviewed the literature on the acral lesions seen in children and adults with COVID-19 infection to offer guidelines for diagnosis and treatment.

Cutis. 2021;107:139-142.

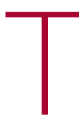

here has been a rise in the prevalence of perniolike lesions-erythematous to violaceous, edematous papules or nodules on the fingers or toes-during the coronavirus disease 2019 (COVID-19) pandemic. These lesions are referred to as "COVID toes." Although several studies have suggested an association with these lesions and COVID-19, and coronavirus particles have been identified in endothelial cells of biopsies of pernio lesions, questions remain on the management, pathophysiology, and implications of these lesions. ${ }^{1}$ We provide a practical review for primary care clinicians and dermatologists on the current management, recommendations, and remaining questions, with particular attention to the distinctions for children vs adults presenting with pernio lesions.

\section{Hypothetical Case of a Child Presenting With Acral Lesions}

A 7-year-old boy presents with acute-onset, violaceous, mildly painful and pruritic macules on the distal toes that began 3 days earlier and have progressed to involve more toes and appear more purpuric. A review of symptoms reveals no fever, cough, fatigue, or viral symptoms. He has been staying at home for the last few weeks with his brother, mother, and father. His father is working in delivery services and is social distancing at work but not at home. His mother is concerned about the lesions, if they could be COVID toes, and if testing is needed for the patient or family. In your assessment and management of this patient, you consider the following questions.

What Is the Relationship Between These Clinical Findings and COVID-19?-Despite negative polymerase chain reaction (PCR) tests reported in cases of chilblains during the COVID-19 pandemic as well as the possibility that these lesions are an indirect result of environmental factors or behavioral changes during quarantine, the majority of studies favor an association between these

\footnotetext{
Ms. Clawson is from Eastern Virginia Medical School, Norfolk. Dr. Tabata is from the Department of Internal Medicine, Massachusetts General Hospital, Boston. Dr. Ko is from the Department of Dermatology, Stanford University, California. 
chilblains lesions and COVID-19 infection. ${ }^{2,3}$ Most compellingly, COVID-19 viral particles have been identified by immunohistochemistry and electron microscopy in the endothelial cells of biopsies of these lesions. ${ }^{1}$ Additionally, there is evidence for possible associations of other viruses, including Epstein-Barr virus and parvovirus B19, with chilblains lesions. ${ }^{4,5}$ In sum, with the lack of any large prospective study, the weight of current evidence suggests that these perniolike skin lesions are not specific markers of infection with severe acute respiratory syndrome coronavirus 2 (SARS-CoV-2). ${ }^{6}$

Published studies differ in reporting the coincidence of perniolike lesions with typical COVID-19 symptoms, including fever, dyspnea, cough, fatigue, myalgia, headache, and anosmia, among others. Some studies have reported that up to $63 \%$ of patients with reported perniolike lesions developed typical COVID-19 symptoms, but other studies found that no patients with these lesions developed symptoms. ${ }^{6-11}$ Studies with younger cohorts tended to report lower prevalence of COVID-19 symptoms, and within cohorts, younger patients tended to have less severe symptoms. For example, $78.8 \%$ of patients in a cohort $(n=58)$ with an average age of 14 years did not experience COVID-19-related symptoms. ${ }^{6}$ Based on these data, it has been hypothesized that patients with chilblainslike lesions may represent a subpopulation who will have a robust interferon response that is protective from more symptomatic and severe COVID-19.12-14

Current evidence suggests that these lesions are most likely to occur between 9 days and 2 months after the onset of COVID-19 symptoms. ${ }^{4,9,10}$ Most cases have been only mildly symptomatic, with an overall favorable prognosis of both lesions and any viral symptoms. ${ }^{8,10}$ The lesions typically resolve without treatment within a few days of initial onset. ${ }^{15,16}$

What Should Be the Workup and Management of These Lesions?-Given the currently available information and favorable prognosis, usually no further workup specific to the perniolike lesions is required in the case of an asymptomatic child presenting with acral lesions, and the majority of management will center around patient and parent/guardian education and reassurance. When asked by the patient's parent, "What does it mean that my child has these lesions?", clinicians can provide information on the possible association with COVID-19 and the excellent, self-resolving prognosis. An example of honest and reasonable phrasing with current understanding might be, "We are currently not certain if COVID-19 causes these lesions, although there are data to suggest that they are associated. There are a lot of data showing that children with these lesions either do not have any symptoms or have very mild symptoms that resolve without treatment."

For management, important considerations include how painful the lesions are to the individual patient and how they affect quality of life. If less severe, clinicians can reassure patients and parents/guardians that the lesions will likely self-resolve without treatment. If worsening or symptomatic, clinicians can try typical treatments for chilblains, such as topical steroids, whole-body warming, and nifedipine. ${ }^{17-19}$ Obtaining a review of symptoms, including COVID-19 symptoms and general viral symptoms, is important given the rare cases of children with severe COVID-19. ${ }^{20,21}$

The question of COVID-19 testing as related to these lesions remains controversial, and currently there are still differing perspectives on the need for biopsy, PCR for COVID-19, or serologies for COVID-19 in patients presenting with these lesions. Some experts report that additional testing is not needed in the pediatric population because of the high frequency of negative testing reported to date. ${ }^{22,23}$ However, these children may be silent carriers, and until more is known about their potential to transmit the virus, testing may be considered if resources allow, particularly if the patient has a known exposure. ${ }^{10,12,16,24}$ The ultimate decision to pursue biopsy or serologic workup for COVID-19 remains up to clinical discretion with consideration of symptoms, severity, and immunocompromised household contacts. If lesions developed after infection, PCR likely will result negative, whereas serologic testing may reveal antibodies.

\section{Hypothetical Case of an Adult Presenting With Acral Lesions and COVID-19 Symptoms}

A 50-year-old man presents with acute-onset, violaceous, painful, edematous plaques on the distal toes that began 3 days earlier and have progressed to include the soles. A review of symptoms reveals fever (temperature, $38.4{ }^{\circ} \mathrm{C}$ $\left.\left[101^{\circ} \mathrm{F}\right]\right)$, cough, dyspnea, diarrhea, and severe asthenia. He has had interactions with a coworker who recently tested positive for COVID-19.

How Should You Consider These Lesions in the Context of the Other Symptoms Concerning for COVID-19?In contrast to the asymptomatic child above, this adult has chilblainslike lesions and viral symptoms. In adults, chilblainslike lesions have been associated with relatively mild COVID-19, and patients with these lesions who are otherwise asymptomatic have largely tested negative for COVID-19 by PCR and serologic antibody testing. ${ }^{11,25,26}$

True acral ischemia, which is more severe and should be differentiated from chilblains, has been reported in critically ill patients. ${ }^{9}$ Additionally, studies have found that retiform purpura is the most common cutaneous finding in patients with severe COVID-19. ${ }^{27}$ For this patient, who has an examination consistent with progressive and severe chilblainslike lesions and suspicion for COVID-19 infection, it is important to observe and monitor these lesions, as clinical progression suggestive of acral ischemia or retiform purpura should be taken seriously and may indicate worsening of the underlying disease. Early intervention with anticoagulation might be considered, though there currently is no evidence of successful treatment. ${ }^{28}$ 
What Causes These Lesions in a Patient With COVID-19?-The underlying pathophysiology has been proposed to be a monocytic-macrophage-induced hyperinflammatory systemic state that damages the lungs, as well as the gastrointestinal, renal, and endothelial systems. The activation of the innate immune system triggers a cytokine storm that creates a hypercoagulable state that ultimately can manifest as superficial thromboses, leading to gangrene of the extremities. Additionally, interferon response and resulting hypercytokinemia may cause direct cytopathic damage to the endothelium of arterioles and capillaries, causing the development of papulovesicular lesions that resemble the chilblainslike lesions observed in children. ${ }^{29}$ In contrast to children, who typically have no or mild COVID-19 symptoms, adults may have a delayed interferon response, which has been proposed to allow for more severe manifestations of infection. ${ }^{12,30}$

How Should an Adult With Perniolike Lesions Be Managed?-Adults with chilblainslike lesions and no other signs or symptoms of COVID-19 infection do not necessarily need be tested for COVID-19, given the reports demonstrating most patients in this clinical situation will have negative PCRs and serologies for antibodies. However, there have been several reports of adults with acro-ischemic skin findings who also had severe COVID-19, with an observed incidence of $23 \%$ in intensive care unit patients with COVID-19. ${ }^{27,28,31,32}$ If there is suspicion of infection with COVID-19, it is advisable to first obtain workup for COVID-19 and other viruses that can cause acral lesions, including EpsteinBarr virus and parvovirus. Other pertinent laboratory tests may include D-dimer, fibrinogen, prothrombin time, activated partial thromboplastin time, antithrombin activity, platelet count, neutrophil count, procalcitonin, triglycerides, ferritin, C-reactive protein, and hemoglobin. For patients with evidence of worsening acro-ischemia, regular monitoring of these values up to several times per week can allow for initiation of vascular intervention, including angiontensin-converting enzyme inhibitors, statins, or antiplatelet drugs. ${ }^{32}$ The presence of antiphospholipid antibodies also has been associated with critically ill patients who develop digit ischemia as part of the sequelae of COVID-19 infection and therefore may act as an important marker for the potential to develop disseminated intravascular coagulation in this patient. ${ }^{33}$ Even if COVID-19 infection is not suspected, a thorough review of systems is important to look for an underlying connective tissue disease, such as systemic lupus erythematosus, which is associated with pernio. Associated symptoms may warrant workup with antinuclear antibodies and other appropriate autoimmune serologies.

If there is any doubt of the diagnosis, the patient is experiencing symptoms from the lesion, or the patient is experiencing other viral symptoms, it is appropriate to biopsy immediately to confirm the diagnosis. Prior studies have identified fibrin clots, angiocentric and eccrinotropic lymphocytic infiltrates, lymphocytic vasculopathy, and papillary dermal edema as the most common features in chilblainslike lesions during the COVID-19 pandemic. ${ }^{9}$

For COVID-19 testing, many studies have revealed adult patients with an acute hypercoagulable state testing positive by SARS-CoV-2 PCR. These same patients also experienced thromboembolic events shortly after testing positive for COVID-19, which suggests that patients with elevated D-dimer and fibrinogen likely will have a viral load that is sufficient to test positive for COVID-19.32,34-36 It is appropriate to test all patients with suspected COVID-19, especially adults who are more likely to experience adverse complications secondary to infection.

This patient experiencing COVID-19 symptoms with signs of acral ischemia is likely to test positive by PCR, and additional testing for serologic antibodies is unlikely to be clinically meaningful in this patient's state. Furthermore, there is little evidence that serology is reliable because of the markedly high levels of both false-negative and false-positive results when using the available antibody testing kits. ${ }^{37}$ The latter evidence makes serology testing of little value for the general population, but particularly for patients with acute COVID-19.

\section{Conclusion and Outstanding Questions}

There is evidence suggesting an association between chilblainslike lesions and COVID-19.11,22,38,39 Children presenting with these lesions have an excellent prognosis and only need a workup or treatment if there are other symptoms, as the lesions self-resolve in the majority of reported cases. ${ }^{7-9}$ Adults presenting with these lesions and without symptoms likewise are unlikely to test positive for COVID-19, and the lesions typically resolve spontaneously or with first-line treatment. However, adults presenting with these lesions and COVID-19 symptoms should raise clinical concern for evolving skin manifestations of acroischemia. If the diagnosis is uncertain or systemic symptoms are concerning, biopsy, COVID-19 PCR, and other appropriate laboratory workup should be obtained.

There remains controversy and uncertainty over the relationship between these skin findings and SARS-CoV-2 infection, with clinical evidence to support both a direct relationship representing convalescent-phase cutaneous reaction as well as an indirect epiphenomenon. If there was a direct relationship, we would have expected to see a rise in the incidence of acral lesions proportionate to the rising caseload of COVID-19 after the reopening of many states in the summer of 2020. Similarly, because young adults represent the largest demographic of increasing cases and as some schools have remained open for in-person instruction during the current academic year, we also would have expected the incidence of chilblainslike lesions presenting to dermatologists and pediatricians to increase alongside these cases. Continued evaluation of emerging literature and ongoing efforts to understand the cause of this observed phenomenon will hopefully help us arrive at a future understanding of the pathophysiology of this puzzling skin manifestation. ${ }^{40}$ 


\section{REFERENCES}

1. Colmenero I, Santonja C, Alonso-Riaño M, et al. SARS-CoV-2 endothelial infection causes COVID-19 chilblains: histopathological, immunohistochemical and ultrastructural study of seven paediatric cases. Br J Dermatol. 2020;183:729-737. doi:10.1111/bjd.19327

2. Neri I, Virdi A, Corsini I, et al. Major cluster of paediatric "true" primary chilblains during the COVID-19 pandemic: a consequence of lifestyle changes due to lockdown. J Eur Acad Dermatol Venereol. 2020;34:26302635. doi:10.1111/jdv.16751

3. Hubiche T, Le Duff F, Chiaverini C, et al. Negative SARS-CoV-2 PCR in patients with chilblain-like lesions [letter]. Lancet Infect Dis. June 18, 2020. doi:10.1016/S1473-3099(20)30518-1

4. Pistorius MA, Blaise S, Le Hello C, et al. Chilblains and COVID19 infection: causality or coincidence? How to proceed? J Med Vasc. 2020;45:221-223. doi:10.1016/j.jdmv.2020.05.002

5. Massey PR, Jones KM. Going viral: a brief history of Chilblain-like skin lesions ("COVID toes") amidst the COVID-19 pandemic. Semin Oncol. 2020;47:330-334. doi:10.1053/j.seminoncol.2020.05.012

6. Docampo-Simón A, Sánchez-Pujol MJ, Juan-Carpena G, et al. Are chilblain-like acral skin lesions really indicative of COVID-19? A prospective study and literature review [letter]. J Eur Acad Dermatol Venereol. 2020;34:e445-e446. doi:10.1111/jdv.16665

7. El Hachem M, Diociaiuti A, Concato C, et al. A clinical, histopathological and laboratory study of 19 consecutive Italian paediatric patients with chilblain-like lesions: lights and shadows on the relationship with COVID-19 infection. J Eur Acad Dermatol Venereol. 2020;34:2620-2629. doi:10.1111/jdv.16682

8. Recalcati S, Barbagallo T, Frasin LA, et al. Acral cutaneous lesions in the time of COVID-19. J Eur Acad Dermatol Venereol. 2020;34:e346-e347. doi:10.1111/jdv.16533

9. Andina D, Noguera-Morel L, Bascuas-Arribas M, et al. Chilblains in children in the setting of COVID-19 pandemic. Pediatr Dermatol. 2020;37:406-411. doi:10.1111/pde.14215

10. Casas CG, Català A, Hernández GC, et al. Classification of the cutaneous manifestations of COVID-19: a rapid prospective nationwide consensus study in Spain with 375 cases. Br J Dermatol. 2020;183:71-77. doi:10.1111/bjd.19163

11. Freeman EE, McMahon DE, Lipoff JB, et al. Pernio-like skin lesions associated with COVID-19: a case series of 318 patients from 8 countries. J Am Acad Dermatol. 2020;83:486-492. doi:10.1016/j.jaad.2020.05.109

12. Kolivras A, Dehavay F, Delplace D, et al. Coronavirus (COVID-19) infection-induced chilblains: a case report with histopathologic findings. JAAD Case Rep. 2020;6:489-492. doi:10.1016/j.jdcr.2020.04.011

13. Damsky W, Peterson D, King B. When interferon tiptoes through COVID-19: pernio-like lesions and their prognostic implications during SARS-CoV-2 infection. I Am Acad Dermatol. 2020;83:E269-E270. doi:10.1016/j.jaad.2020.06.052

14. Lipsker D. A chilblain epidemic during the COVID-19 pandemic. A sign of natural resistance to SARS-CoV-2? Med Hypotheses. 2020;144:109959. doi:10.1016/j.mehy.2020.109959

15. Kaya G, Kaya A, Saurat J-H. Clinical and histopathological features and potential pathological mechanisms of skin lesions in COVID-19: review of the literature. Dermatopathology. 2020;7:3-16. doi:10.3390/ dermatopathology7010002

16. Pavone P, Marino S, Marino L, et al. Chilblains-like lesions and SARS-CoV-2 in children: An overview in therapeutic approach. Dermatol Ther. 2021;34:E14502. doi:https://doi.org/10.1111/dth.14502

17. Dowd PM, Rustin MH, Lanigan S. Nifedipine in the treatment of chilblains. Br Med J (Clin Res Ed). 1986;293:923-924. doi:10.1136/ bmj.293.6552.923-a

18. Rustin MH, Newton JA, Smith NP, et al. The treatment of chilblains with nifedipine: the results of a pilot study, a double-blind placebocontrolled randomized study and a long-term open trial. Br J Dermatol. 1989;120:267-275. doi:10.1111/j.1365-2133.1989.tb07792.x

19. Almahameed A, Pinto DS. Pernio (chilblains). Curr Treat Options Cardiovasc Med. 2008;10:128-135. doi:10.1007/s11936-008-0014-0

20. Chen F, Liu ZS, Zhang FR, et al. First case of severe childhood novel coronavirus pneumonia in China [in Chinese]. Zhonghua Er Ke
Za Zhi. 2020;58:179-182. doi:10.3760/cma.j.issn.0578-1310 .2020.03.003

21. Choi S-H, Kim HW, Kang J-M, et al. Epidemiology and clinical features of coronavirus disease 2019 in children. Clin Exp Pediatr. 2020; 63:125-132. doi:10.3345/cep.2020.00535

22. Piccolo V, Neri I, Manunza F, et al. Chilblain-like lesions during the COVID-19 pandemic: should we really worry? Int J Dermatol. 2020;59:1026-1027. doi:10.1111/ijd.14993

23. Roca-Ginés J, Torres-Navarro I, Sánchez-Arráez J, et al. Assessment of acute acral lesions in a case series of children and adolescents during the COVID-19 pandemic. JAMA Dermatol. 2020;156:992-997. doi:10.1001/jamadermatol.2020.2340

24. Landa N, Mendieta-Eckert M, Fonda-Pascual P, et al. Chilblain-like lesions on feet and hands during the COVID-19 pandemic. Int J Dermatol. 2020;59:739-743. doi:10.1111/ijd.14937

25. Herman A, Peeters C, Verroken A, et al. Evaluation of chilblains as a manifestation of the COVID-19 pandemic. JAMA Dermatol. 2020;156:998-1003. doi:10.1001/jamadermatol.2020.2368

26. Daneshjou R, Rana J, Dickman M, et al. Pernio-like eruption associated with COVID-19 in skin of color. JAAD Case Rep. 2020;6:892-897. doi:10.1016/j.jdcr.2020.07.009

27. Freeman EE, McMahon DE, Lipoff JB, et al. The spectrum of COVID-19-associated dermatologic manifestations: an international registry of 716 patients from 31 countries. J Am Acad Dermatol. 2020;83:1118-1129. doi:10.1016/j.jaad.2020.06.1016

28. Zhang $\mathrm{Y}, \mathrm{Cao} \mathrm{W}$, Xiao M, et al. Clinical and coagulation characteristics of 7 patients with critical COVID-2019 pneumonia and acro-ischemia [in Chinese]. Zhonghua Xue Ye Xue Za Zhi. 2020;41:E006. doi:10.3760/ cma.j.issn.0253-2727.2020.0006

29. Criado PR, Abdalla BMZ, de Assis IC, et al. Are the cutaneous manifestations during or due to SARS-CoV-2 infection/COVID-19 frequent or not? revision of possible pathophysiologic mechanisms. Inflamm Res. 2020;69:745-756. doi:10.1007/s00011-020-01370-w

30. Park A, Iwasaki A. Type I and type III interferons-induction, signaling, evasion, and application to combat COVID-19. Cell Host Microbe. 2020;27:870-878. doi:10.1016/j.chom.2020.05.008

31. Wollina U, Karadağ AS, Rowland-Payne C, et al. Cutaneous signs in COVID-19 patients: a review. Dermatol Ther. 2020;33:E13549. doi:10.1111/dth.13549

32. Alonso MN, Mata-Forte T, García-León N, et al. Incidence, characteristics, laboratory findings and outcomes in acro-ischemia in COVID-19 patients. Vasc Health Risk Manag. 2020;16:467-478. doi:10.2147/VHRM. S276530

33. Zhang L, Yan X, Fan Q, et al. D-dimer levels on admission to predict in-hospital mortality in patients with COVID-19. J Thromb Haemost. 2020;18:1324-1329. doi:10.1111/jth.14859

34. Helms J, Tacquard C, Severac F, et al. High risk of thrombosis in patients with severe SARS-CoV-2 infection: a multicenter prospective cohort study. Intensive Care Med. 2020;46:1089-1098. doi:10.1007/s00134-020-06062-x

35. Barton LM, Duval EJ, Stroberg E, et al. COVID-19 autopsies, Oklahoma, USA. Am J Clin Pathol. 2020;153:725-733. doi:10.1093/ajcp/aqaa062

36. Wichmann D, Sperhake J-P, Lütgehetmann M, et al. Autopsy findings and venous thromboembolism in patients with COVID-19. Ann Intern Med. 2020;173:268-277. doi:10.7326/M20-2003

37. Bastos ML, Tavaziva G, Abidi SK, et al. Diagnostic accuracy of serological tests for COVID-19: systematic review and meta-analysis. BMJ. 2020;370:m2516. doi:10.1136/bmj.m2516

38. Galván Casas C, Català A, Carretero Hernández G, et al. Classification of the cutaneous manifestations of COVID-19: a rapid prospective nationwide consensus study in Spain with 375 cases. $\mathrm{Br} J$ Dermatol. 2020;183:71-77. doi:10.1111/bjd.19163

39. Fernandez-Nieto D, Jimenez-Cauhe J, Suarez-Valle A, et al. Characterization of acute acral skin lesions in nonhospitalized patients: a case series of 132 patients during the COVID-19 outbreak. J Am Acad Dermatol. 2020;83:E61-E63. doi:10.1016/j.jaad.2020.04.093

40. Deutsch A, Blasiak R, Keyes A, et al. COVID toes: phenomenon or epiphenomenon? J Am Acad Dermatol. 2020;83:E347-E348. doi:10.1016/j. jaad.2020.07.037 